\title{
Proposal and design of Airy-based rocket pulses for invariant propagation in lossy dispersive media
}

\author{
Miguel A. Preciado* and Kate Sugden \\ Aston Institute of Photonic Technologies, Aston University, Birmingham B4 7ET, UK \\ ${ }^{*}$ Corresponding author: m.preciado@aston.ac.uk
}

Received September 19, 2012; revised October 31, 2012; accepted November 1, 2012; posted November 2, 2012 (Doc. ID 176570); published November 29, 2012

\begin{abstract}
A novel (to our knowledge) kind of Airy-based pulse with an invariant propagation in lossy dispersive media is proposed. The basic principle is based on an optical energy trade-off between different parts of the pulse caused by the chromatic dispersion, which is used to compensate the attenuation losses of the propagation medium. Although the ideal concept of the proposed pulses implies infinite pulse energy, the numerical simulations show that practical finite energy pulses can be designed to obtain a partially invariant propagation over a finite distance of propagation. (c) 2012 Optical Society of America

OCIS codes: $\quad 060.0060,260.2030,320.0320$.
\end{abstract}

Ideal Airy pulses have an infinite energy, which allows them to propagate undistorted in a dispersive medium. They were proposed in 1979 [1] within the context of quantum mechanics but have recently become topical in optical systems. Due to the unique properties of Airy pulses, the intensity profile remains invariant during propagation. Spatiotemporal light bullet Airy pulses have recently been investigated under linear and nonlinear conditions $[2, \underline{3}]$ and have also been proposed in a patented optical pulses transmission system in [4]. Although the intensity profile remains proportionally constant, typical Airy pulses are still affected by the attenuation of the media, decreasing the peak pulse intensity levels. In this Letter, a novel (to our knowledge) kind of Airy-based pulse that exhibits invariance to both the attenuation and chromatic dispersion introduced by a lossy dispersive medium is proposed. This unique combination of properties is obtained by an optical energy trade-off, where some parts of the pulse feed the pulse peak region with optical energy to compensate for the losses due to the pulse propagation, in a similar way that a rocket uses its propulsion system energy in order to compensate the energy losses in the rocket propagation.

In the remainder of this Letter, the theoretical basis of the proposed Airy rocket pulse propagation invariance is shown, and an example is designed and numerically simulated. Finally, we summarize and conclude our work.

A dispersive lossy medium can be characterized by its spectral response, which can be modeled as $H(\omega, z)=$ $\exp (-z(\alpha(\omega)+j \beta(\omega)))$, where $\omega$ is the baseband angular frequency, i.e., $\omega=\omega_{\text {opt }}-\omega_{0}, \omega_{\text {opt }}$ is the optical angular frequency, $\omega_{0}$ is the central angular frequency, $j$ is the imaginary unit, $\alpha(\omega)$ represents the attenuation, and $\beta(\omega)$ is the propagation constant of the single mode as a function of $\omega . \beta(\omega)$ can be approximated as a Taylor expansion until the second order of $\omega, \beta(\omega)=\beta_{0}+\beta_{1} \omega+$ $\left(\beta_{2} / 2\right) \omega^{2}$, where $\beta_{i}=d^{i} \beta(\omega) / d \omega^{i}$ at $\omega=0$.

An ideal Airy pulse can be defined from its spectral function, $A(\omega)=\exp \left(j \xi \omega^{3}\right)$ [5]. In the absence of attenuation, an ideal Airy pulse propagates in a dispersive media with an invariant waveform. However, when attenuation is considered, there is a decrease in the temporal intensity of the pulse as it propagates through the medium.
Here we propose a novel kind of Airy-based "rocket" pulse, which exhibits not only dispersion invariance but also attenuation invariance in the peak power as it propagates. The proposed "Airy rocket" pulse can be defined from its spectral function:

$$
A_{r}(\omega)=A(\omega) G(\omega)=\exp \left(\zeta \omega+j \xi \omega^{3}\right),
$$

where $\zeta$ is a real constant number and $G(\omega)=\exp (\zeta \omega)$ modifies the spectral amplitude of the Airy pulse $A(\omega)$. The propagated pulse spectral function at a position $z$ can be calculated by applying the spectral response $H(\omega, z)$, obtaining $F_{\text {prob }}(\omega, z)=A_{r}(\omega) H(\omega, z)$, which can be expressed as

$$
F_{\text {prob }}(\omega, z)=\exp \left(\zeta \omega-\alpha z+j\left(\xi \omega^{3}-\beta(\omega) z\right)\right),
$$

where the attenuation can be considered to be approximately constant provided $\exp ((\alpha(\omega)-\alpha) z) \approx 1$ over the whole bandwidth of interest and propagation distance range, with $\alpha=\alpha(0)$. By applying some algebraic manipulation, we can find that

$$
\begin{aligned}
F_{\text {prop }}(\omega, z)= & \exp (\zeta \omega-\alpha z) \\
& \exp \left(j \xi(\omega-\Delta \omega(z))^{3}-j(\phi(z)+\Delta t(z) \omega)\right) \\
= & \exp \left(\zeta(\omega-\Delta \omega(z))+j \xi(\omega-\Delta \omega(z))^{3}\right) \\
& \exp (\zeta \Delta \omega(z)-\alpha z-j(\phi(z)+\Delta t(z) \omega)) \\
= & A_{r}(\omega-\Delta \omega(z)) \exp (\zeta \Delta \omega(z) \\
& -\alpha z-j(\phi(z)+\Delta t(z) \omega)),
\end{aligned}
$$

where $\phi(z)=\beta_{0} z-\xi \Delta \omega(z)^{3}$,

$$
\begin{aligned}
\Delta \omega(z) & =\beta_{2} z / 6 \xi \\
\Delta t(z) & =3 \xi \Delta \omega(z)^{2}+\beta_{1} z, \\
\xi \omega^{3}-\beta(\omega) z & =\xi(\omega-\Delta \omega(z))^{3}-\phi(z)-\Delta t(z) \omega,
\end{aligned}
$$

which can be easily deduced by expressing the $\beta(\omega)$ and $(\omega-\Delta \omega(z))^{3}$ terms in their polynomial expanded form. 
From Eq. (3) we can observe that the effect of the attenuation is cancelled if $\zeta \Delta \omega(z)-\alpha z=0$, thereby obtaining

$$
\zeta=6 \xi \alpha / \beta_{2}
$$

and finally, we have the expression of the propagated pulse as

$$
F_{\text {prop }}(\omega, z)=A_{r}(\omega-\Delta \omega(z)) \exp (-j(\phi(z)+\Delta t(z) \omega)) .
$$

In the time domain, we can obtain the temporal complex envelope function of the propagated pulse $f_{\text {prop }}(t, z)$ by applying the inverse Fourier transform to Eq. (8) such that

$$
f_{\text {prop }}(t, z)=a_{r}(t-\Delta t(z)) \exp (j(\Delta \omega(z) t-\phi(z))),
$$

where $a_{r}(t)$ is the complex envelope of the Airy rocket pulse, which can be obtained from the inverse Fourier transform of $A_{r}(\omega)$. We finally obtain from Eq. (9) the invariance of the temporal intensity of the Airy rocket pulse as it propagates in a dispersive and lossy media:

$$
\left|f_{\text {prop }}(t, z)\right|^{2}=\left|a_{r}(t-\Delta t(z))\right|^{2} .
$$

Like the original Airy pulse, the ideal Airy rocket pulse has infinite energy; however, this energy needs to be truncated in order to obtain a practical finite-energy pulse. Here, a spectral truncation using a spectral flattop window function $W(\omega)$ is proposed such that

$$
A_{r, W}(\omega)=A(\omega) G(\omega) W(\omega),
$$

where $W(\omega)$ must be approximately constant over most of the total window bandwidth, $B$, in order not to distort the spectral components of $A_{r}(\omega)$ in $A_{r, W}(\omega)$.

In order to show the invariant propagation of the finite energy Airy rocket pulse, we design and numerically simulate an example of pulse propagation in a singlemode fiber. Without loss of generality, in this example we assume a standard single-mode fiber and ITU-T G.652 specifications, and this dispersion parameter can be modeled as $D(\lambda)=D_{1550}+S_{1550}\left(\lambda-\lambda_{0}\right) \mathrm{ps} /(\mathrm{nm} \cdot \mathrm{Km})$, where $\lambda_{0}=1550 \mathrm{~nm}, \quad D_{1550}=17 \mathrm{ps} /(\mathrm{nm} \cdot \mathrm{Km})$ and $S_{1550}=0.056 \mathrm{ps} /\left(\mathrm{nm}^{2} \cdot \mathrm{Km}\right)$. From this we can obtain values for $\beta_{2}=-21.68 \mathrm{ps}^{2} / \mathrm{km}$ and $\beta_{3}=0.0911 \mathrm{ps}^{2} / \mathrm{km}$ at the central frequency $\omega_{0}=2 \pi f_{0}$ with $f_{0}=193.413 \mathrm{THz}$ (or $1550 \mathrm{~nm}$ wavelength). The attenuation parameter is modeled as $\alpha(\lambda)=0.2-0.00035\left(\lambda-\lambda_{0}\right)$, where the slope has been calculated using typical attenuation values in Table 1.2 of [] $]$, assuming a small bandwidth around $\lambda_{0}$ In our design we approximate $\alpha(\lambda) \approx \alpha=0.2 \mathrm{~dB} / \mathrm{km}$. Nonlinear effects are not involved in the invariant propagation of the proposed pulse and are out of the scope of the present work. The initial finite energy Airy rocket pulse to be launched into the optical fiber can be defined by its spectral function $A_{r . W}(\omega)$ using Eq. (11), where we assume a total bandwidth of $B=0.2 \mathrm{THz}(1.6 \mathrm{~nm}$ wavelength bandwidth). The function $A(\omega)$ is defined by the design parameter $\xi=301.9 \mathrm{ps}^{3}$, corresponding

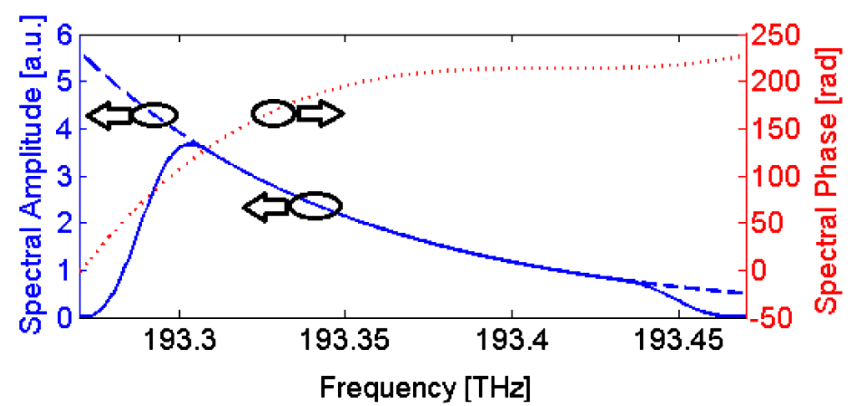

Fig. 1. (Color online) Spectral amplitude of the ideal infinite energy Airy rocket pulse (blue dashed curve and the finite energy pulse (blue solid curve), and spectral phase (red dotted curve) for both.

to a frequency shift per distance $\Delta \omega(z) / z \approx 1 \mathrm{GHz} / \mathrm{km}$, where Eq. (4) is used. The selected window function $W(\omega)$ is a raised cosine function with a roll-off factor of $40 \%$, with a total bandwidth of $B$, centered at 193.370 THz. The resulting spectral function $A_{r, w}(\omega)$ of the designed initial pulse is shown in Fig. 1. There are several pulse-shaping techniques that can be applied to generate the designed pulse. Fiber Bragg gratings [ [7] have proved to be useful in shaping optical pulses of relative complexity. Another well-known pulse-shaping technique is based on spatial light modulators [8], which are especially suited for our purpose, taking into account the simplicity of the required pulse spectral function, since they can shape almost arbitrary optical pulses by optical manipulation of the optical signal directly in the spectral domain.

The propagation of the pulse is numerically simulated along $70 \mathrm{~km}$ of single-mode fiber. Figure $\underline{2}$ shows the

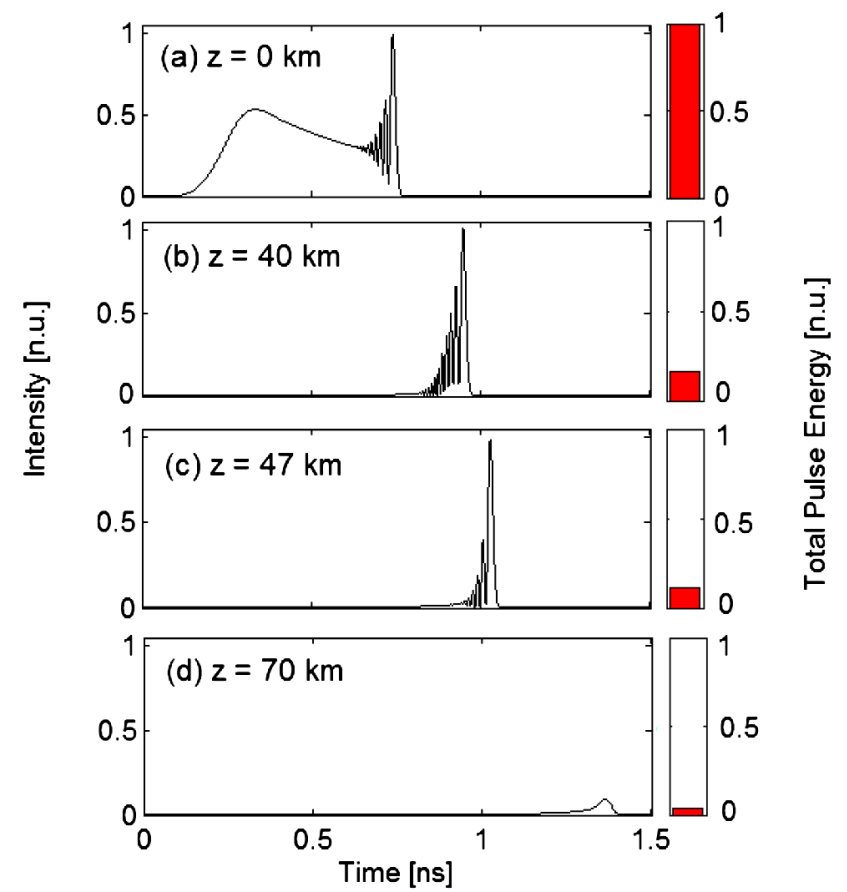

Fig. 2. (Color online) Temporal intensity of the propagation of the Airy rocket pulse designed in the example in a path of $70 \mathrm{~km}$, where the corresponding total pulse energy is indicated in the right-hand side (Media 1). Four points of the path shown: (a) $z=0 \mathrm{~km}$, (b) $z=40 \mathrm{~km}$, (c) $z=47 \mathrm{~km}$, and (d) $z=70 \mathrm{~km}$. 


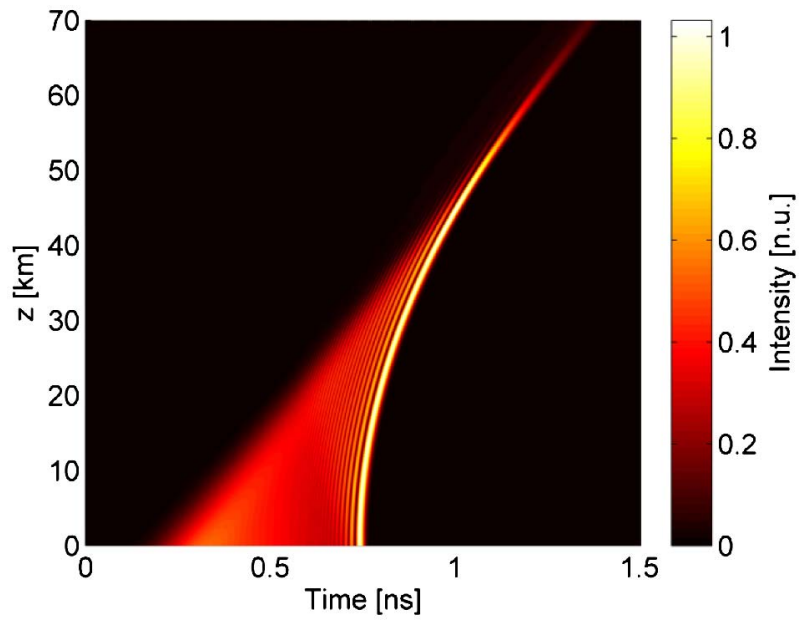

Fig. 3. (Color online) Color map representation of the evolution of the temporal intensity of the propagated Airy rocket pulse designed in the example in a path of $70 \mathrm{~km}$, as it propagates in $z$.

temporal intensity profile and the total pulse energy at four points of the fiber, and corresponding Media 1 shows the representation of the propagation along the whole path. Additionally, in Fig. $\underline{3}$, the pulse propagation is shown in a two-dimensional color map representation of the temporal intensity of the propagated pulse as a function of $t$ and $z$. As can be observed, the attenuation invariance property partially remains in the head of the finite energy Airy rocket pulse. As the distance increases, a decreasing portion of the pulse remains invariant until a distance of $z \sim 47 \mathrm{~km}$. It is worth noting that the wide initial pulse width at $z=0$ of this design may make this approach impractical for optical communications applications. However, since the pulse gets narrower as it propagates, the initial pulse width can be narrowed by adding linear dispersion, obtaining better spectral efficiency in such applications. If noise added to the initial pulse is considered, it can be seen that only linear effects are involved in the propagation of this pulse. The noise will be present as an additive noise and will be dispersed and linearly summed with the propagated pulse.

Figure 4 shows the maximum intensity peak and the total energy of the pulse as a function of $z$. Although the total pulse energy is attenuated and exponentially decreases, the pulse intensity peak remains constant over a distance of approximately of $z \sim 47 \mathrm{~km}$. This effect can be interpreted as a local self-amplification effect, where the intensity peak of the pulse is fed by the optical energy from the other parts of the pulse, which

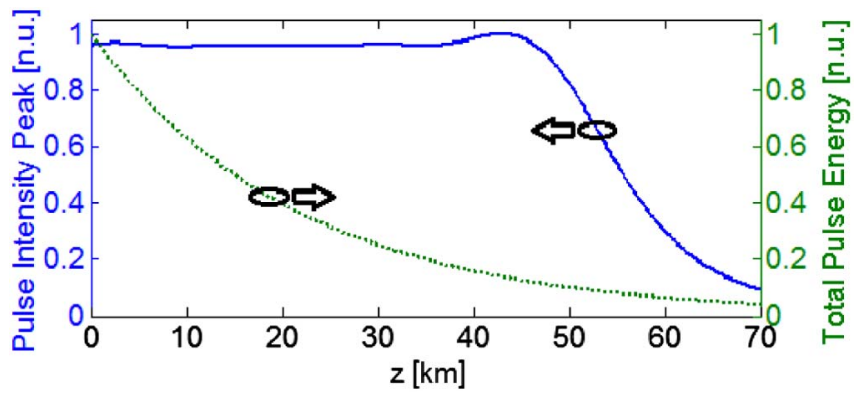

Fig. 4. (Color online) Pulse intensity peak (blue solid curve) and total pulse energy (green dotted curve).

are transferred by the chromatic dispersion. At some point, the intensity peak unavoidably decreases, since the total available pulse energy is finite, illustrating the "rocketlike" behavior of the proposed pulse. It is worth noting that the wavelength dependence of the attenuation parameter has a very marginal effect in the simulation results and can be considered negligible in this example.

In conclusion, in this Letter we have presented a novel kind of Airy-based pulse with invariance to both attenuation and chromatic dispersion of a lossy dispersive media. We have showed that this property partially remains in the designed practical finite energy pulse obtained. It is worth noting that although an optical fiber has been chosen as the propagation media in the example, these pulses can designed for and applied to any kind of lossy dispersive media. The pulse shape can be optimized for propagation over different distances or fiber types.

This research was supported by a Marie Curie Intra European Fellowship within the Seventh European Community Framework Programme.

\section{References}

1. M. V. Berry and N. L. Balazs, Am. J. Phys. 47, 264 (1979).

2. A. Chong, W. H. Renninger, D. N. Christodoulides, and F. W. Wise, Nat. Photonics 4, 103 (2010).

3. D. Abdollahpour, S. Suntsov, D. G. Papazoglou, and S. Tzortzakis, Phys. Rev. Lett. 105, 253901 (2010).

4. M. A. Preciado and M. A. Muriel, "Método y sistema para la transmisión de pulsos ópticos a través de medios dispersivos," Spanish patent ES 2364935 B2 (February 14, 2012).

5. O. Vallée and M. Soares, Airy Functions and Applications to Physics (Imperial College, 2004), Chap. 4.

6. ITU-T, Optical Fibres, Cables and Systems (ITU, 2009) Chap. 7.

7. M. Ibsen and R. Feced, Opt. Lett. 28, 980 (2003).

8. A. M. Weiner, Prog. Quantum Electron. 19, 161 (1995). 